\title{
Principal-Agent Theory Based Risk Allocation Model for Virtual Enterprise
}

\author{
Min Huang ${ }^{1}$, Guike Chen ${ }^{1 *}$, Wai-Ki Ching ${ }^{2}$, Tak Kuen Siu ${ }^{3}$ \\ ${ }^{1}$ College of Information Science and Engineering, Northeastern University, Key Laboratory of Integrated Automation of Process Industry \\ (Northeastern University), Ministry of Education, Shenyang, China; ${ }^{2}$ Department of Mathematics, The University of Hong Kong, \\ Hong Kong, China; ${ }^{3}$ Department of Actuarial Studies, Faculty of Business and Economics, Macquarie University, Sydney, Australia. \\ Email: mhuang@mail.neu.edu.cn, guikechen@sina.com.cn
}

Received February $3^{\text {rd }}, 2010$; revised March 19 ${ }^{\text {th }}, 2010$; accepted April $29^{\text {th }}, 2010$.

\begin{abstract}
In this paper, we consider a risk analysis model for Virtual Enterprise (VE) by exploring the state of the art of the principal-agent theory. In particular, we deal with the problem of allocating the cost of risk between two parties in a VE, namely, the owner and the partner(s). We first consider the case of a single partner of VE with symmetric information or asymmetric information and then the case of multiple partners. We also build a model for the optimal contract of the risk allocation based on the principal-agent theory and analyze it through specific example. At last we consider the case of multiple principal with potentially many partners based on common agency.
\end{abstract}

Keywords: Virtual Enterprise, Risk Allocation, Principal-Agent Theory, Risk Aversion, Common Agency

\section{Introduction}

Virtual Enterprise (VE) is a dynamic alliance composed of independent individual enterprises which locate in different area. It's designed to adapt to rapidly changing market opportunities, so as to achieve the sharing of skills, core competencies and resources [1,2]. Based on this concept, on one hand, member enterprises in a VE, which are geographically distributed, keep their independence and autonomy. On the other hand, they provide their own core competencies in different areas such as marketing, engineering and manufacturing to the VE. When new market requirements arise and individual enterprises do not have all necessary skills and competencies to undertake these requirements independently, by combining specific expertise of other enterprises, it is possible to create a VE which is capable of responding to the new requirements. In a certain sense, the essence of VE has its basis in an early and fundamental concept of economics, namely, the division of labor, which has its origin in the classics, namely, the wealth of nations, by Adam Smith first published in 1776.

In spite of substantial advantages of VE, there are lots of risks associated with it, these risks include investment risk [3], operation risk [4], moral hazard [5,6] and market risk, and so on. These incomplete nesses arises from member enterprises not having sufficient background information about the other member enterprises or about market environment in which the VE has to operate. The investigation of the structure, operations and economic implications of a VE has received much interest among researchers in the field. Much attention has been paid on some aspects of VE, such as partner selection [7,8], operation management [9], information exchanges [10] and their scales. However, an important issue, the risk management of VE, has not been well-explored and addressed until recently. Since virtual enterprises (VEs) are profit driven, it is one of the key issues to the successful running of VEs whether they could construct reasonable and efficient risk allocation mechanism in the operation process to prevent some members from gaining profit by harming others. The establishment of a VE can not reduce or eliminate the risk due to the uncertainty of market opportunities and production capacities. The risk of the whole enterprise is re-distributed among different members in the VE. There are some ways to mitigate the risk in the cooperation process, such as partner selection $[11,12]$, cooperation contract design [12], and coordination mechanism design [13]. After reviewing the related literature, we found out that researchers have carried out certain publications on VE's risk.

Based on the research of risk in supply chain [14], it produces partnerships [6] and joint ventures [15]. We consider a risk allocation model for VE by exploring the state of the art of the principal-agent theory. In particular, we deal with the problem of allocating the cost of risk 
between two parties in a VE, namely, the owner and the partners. Our analysis invokes some basic and important concepts for the risk analysis, including utility function, risk-aversion level, principal-agent theory [16] and common agency $[17,18]$. Here we first deal with the case of a single partner of VE with symmetric information or asymmetric information. Then, the model is extended to deal with the case of multiple partners. We also build a model for the optimal contract of the risk allocation based on the principal-agent theory. At last we extend the principal-agent framework with risk-neutral principals to situations in which several principals simultaneously and independently attempt to influence a common agent. The remainder of this paper is organized as follows. In Section 2, we give a brief discussion on some basic concepts of risk analysis and related assumptions. In Section 3, we present our risk allocation models. In Section 4, a specific example is given to demonstrate our models in section 3. In Section 5, we discuss the incentive mechanism on the basis of common agency $[18,19]$ when the relationships between the principals is competition. Finally concluding remarks are given in Section 6.

\section{Basic Concepts and Assumptions}

In this section, we provide a brief discussion on some basic concepts of risk analysis, namely, the utility function, the risk aversion and the principal-agent theory, in the context of VE which involving an owner and $n$ risk-averse member enterprises (partners). These concepts also play fundamental and important role in financial economics and corporate finance. Then summarize the major notations to be used in this paper and give the assumptions.

First of all, utility can be considered as goods or services that meet the needs of consumer's ability or desire. The utility function is defined as a mapping function which maps goods or services to consumer preferences. Let $x$ denote the receipts or earnings of a member enterprise. Then, the utility function is given by $\mu(x)$, which is interpreted as goods or services that meet the member enterprise's preference. It is a representation of the member attitude towards risk.

The degree of risk aversion is an important characterization of a utility function. To measure the degree of risk aversion, Arrow (1970) and Pratt (1964) introduce the celebrated Arrow-Pratt ratio of risk aversion level given as follows.

$$
\rho(\omega)=-\frac{\mu^{\prime \prime}(\omega)}{\mu^{\prime}(\omega)}
$$

Principal-agent theory tries to model the following types of questions. One participant (principal) wants to participate in another person (agent) in accordance with the interests of his choice of action, but the principal can not observe directly the agent's actions. What can only be observed are some other variables? These variables are decided by the agent's action and other random factors. The principal's problem is how to incentives the agents in accordance with the information observed to encourage their agents to choose the most favorable actions. The principal-agent model is built to analyze the optimal contract with asymmetric information. To solve the problem conveniently, we consider the optimal contract with symmetric information. The central issue of principal-agent relationship is the alternating between insurance and incentive.

To facilitate our discussion, we define the following notations and impose the following assumptions:

$a$, partner's manpower contributing to the project (the productive effort of the partner);

$\theta=\sum_{i=1}^{r} t_{i} \theta_{i}$, the random variables that not be controlled by the alliance, where $\theta_{1}, \theta_{2}, \ldots, \theta_{r}$ are independent risk factors;

$\sigma^{2}$, the variance of $\theta$;

$g(\theta), G(\theta)$, the probability density function and the distribution function of $\theta$, respectively;

$\pi(a, \theta)$, the monetary income (outputs) of the alliance;

$f(\pi, a)$, the probability density function of $\pi$;

$s(x)$, the incentive contract (a way to repay partner);

$v(x), u(x)$, the owner's and partner's utility function respectively;

$\bar{u}$, the reservations utility (the greatest utility that partners do not accept the contract);

$\rho_{P}$, the owner's risk aversion level;

$\rho_{A}$, the partner's risk aversion level;

$C(a)$, cost function of the effort $a$.

In this paper, we consider an owner and several member enterprises (partners) in a VE. Each partner chooses a level of productive effort $a \geq 0$ and a level of risk aversion $\rho$. Both productive effort $a$ and risk aversion level $\rho$ are individually costly to partners and we assume that the two actions are stochastically independent and the cost of actions can be expressed in monetary units.

\section{The Risk Allocation Models}

In this section, we present the risk allocation model under the assumptions in section 2 based on principal-agent theory which involving an owner and one or $n$ riskaverse partners. We first deal with the case of a single partner of VE with symmetric information and asymmetric information (hidden action) respectively. Then, the 
model is extended to deal with the case of multiple partners.

\subsection{The Optimal Contract of Risk Allocation with Symmetric Information to a Single Partner}

In this subsection, we consider the case that the owner can observe the partner's action (the productive effort) involving an owner and a risk-averse partner in a VE. As the partner's action can be observed, the owner can force the partner to choose the ideal productive effort, so the incentive is surplus.

The risk allocation model is given as follows: Give a $a$, the output is a simple random variable; the owner's objective is to maximize the utility of its own profit by allocating the total revenue from the VE project including choosing $s(\pi)$ :

$$
\begin{aligned}
& \max _{s(\pi)}\left\{E(v(\pi-s(\pi)))=\int v(\pi-s(\pi)) f(\pi, a) d \pi\right\} \\
& \text { s.t. }(I R) \int u(s(\pi)) f(\pi, a) d \pi-C(a) \\
& =E(u(s(\pi)))-C(a) \geq \bar{u}
\end{aligned}
$$

Equation (2) is the partner's individual rationality constraint (IR). We then construct the Lagrange function as below:

$$
\begin{aligned}
L(s(\pi))= & \int v(\pi-s(\pi)) f(\pi, a) d \pi+ \\
& \lambda\left(\int u(s(\pi)) f(\pi, a) d \pi-C(a)-\bar{u}\right)
\end{aligned}
$$

The partial derivative of the function with respect to $s(\pi)$ is given by

$$
-v^{\prime}\left(\pi-s^{*}(\pi)\right)+\lambda u^{\prime}\left(s^{*}(\pi)\right)=0
$$

Therefore, we have

$$
\lambda=\frac{v^{\prime}\left(\pi-s^{*}(\pi)\right)}{u^{\prime}\left(s^{*}(\pi)\right)}
$$

The Lagrange multiplier is a strictly positive constant in (5) (because (2) strictly satisfied). The corresponding optimal condition shows that the ratio of marginal utility of income of the owner and partner is a constant, no relation with the output and uncertain variables $\theta$.

The optimal condition of (5) implicitly defined the optimal contract $s^{*}(\pi)$, from implicit function theorem, the partial derivative with respect to $\pi$ is:

$$
-v^{\prime \prime}\left(1-\frac{d s^{*}}{d \pi}\right)+\lambda u^{\prime \prime} \frac{d s^{*}}{d \pi}=0
$$

Combining the above equations, we get

$$
\frac{d s^{*}}{d \pi}=\frac{\rho_{p}}{\rho_{A}+\rho_{p}}
$$

where $\rho_{P}=-\frac{v^{\prime \prime}}{v^{\prime}}$ and $\rho_{A}=-\frac{u^{\prime \prime}}{u^{\prime}}$

$$
\text { Let } \quad \frac{d s^{*}}{d \pi}=\frac{\rho_{P}}{\rho_{A}+\rho_{P}}=\beta(\pi)
$$

Then we have $s^{*}(\pi)=\alpha+\int_{0}^{\pi} \beta(t) d t$

In particular, if $\rho_{P}$ and $\rho_{A}$ are constants (no relation among their level of income), then the optimal contract is linear, i.e.

$$
s^{*}(\pi)=\alpha+\beta \pi
$$

We define $\triangle R C$ to be the risk cost of the alliance project. Now, the improved risk programming model is given as follows:

$$
\min _{\beta}\left\{\Delta R C=\frac{1}{2} \rho_{A} \sigma^{2} \beta^{2}\right\}
$$

Such that

$$
\begin{aligned}
& \int u(s(\pi)) f(\pi, a) d \pi-C(a) \\
& =E(u(s(\pi)))-C(a) \geq \bar{u} \quad \Delta R C<R \\
& \beta=\arg \max \{E(v(\pi-s(\pi)))\}
\end{aligned}
$$

\subsection{The Optimal Contract of Risk Allocation with Asymmetric Information to a Single Partner}

In this subsection, we consider the case that the owner can't observe the partner's action (the productive effort) involving an owner and a risk-averse partner in a VE. As the partner's action is hidden, the owner has to incentive the partner to choose the ideal productive effort, i.e., the partner chooses action $a$ to maximize the utility of its own profit, where the owner cannot observe the value of $a$. We seek for maximizing the partner's expected utility:

$$
\max _{a}\left\{\int u(s(\pi)) f(\pi, a) d \pi-C(a)\right\}
$$

Equation (12) is the incentive compatibility constraints (IC). The partial derivative with respect to $a$ is:

$$
\int u(s(\pi)) f_{a}(\pi, a) d \pi-C^{\prime}(a)=0
$$

i.e., IC constraint can be replaced by the first-order approach of (13). We then consider maximizing the utility of the owner's profit:

$$
\max _{s(\pi)}\left\{E(v(\pi-s(\pi)))=\int v(\pi-s(\pi)) f(\pi, a) d \pi\right\}
$$


Such that

$$
\begin{array}{r}
\int u(s(\pi)) f(\pi, a) d \pi-C(a) \\
=E(u(s(\pi)))-C(a) \geq \bar{u}
\end{array}
$$

and

$$
\int u(s(\pi)) f_{a}(\pi, a) d \pi=C^{\prime}(a)
$$

Now, we construct the Lagrange function:

$$
\begin{aligned}
L(s(\pi)) & =\int v(\pi-s(\pi)) f(\pi, a) d \pi \\
& +\lambda\left(\int u(s(\pi)) f(\pi, a) d \pi-C(a)-\bar{u}\right) \\
& +\mu\left(\int u(s(\pi)) f_{a}(\pi, a) d \pi-C^{\prime}(a)\right)
\end{aligned}
$$

where $\lambda$ and $\mu$ are the Lagrange multipliers of participation constraint and the incentive constraint, respectively. The optimal condition is given as follows:

$$
\frac{v^{\prime}\left(\pi-s^{*}(\pi)\right)}{u^{\prime}\left(s^{*}(\pi)\right)}=\lambda+\mu \frac{f_{a}(\pi, a)}{f(\pi, a)}
$$

By comparing with (5), it shows that, if the owner cannot observe $a$, the Pareto efficiency risk allocation is impossible. As $\mu>0$ (Holmstrom proved in 1979), in order to motivate the partner to work hard, it has to bare more risks.

\subsection{The Optimal Contract of Risk Allocation with Symmetric Information to Multiple Partners}

In this subsection, we discuss the case of symmetric information with multiple partners that the owner can observe the partner's action (the productive effort) involving an owner and $n$ risk-averse partners in a VE. We first define the following notations and assumptions.

$i$, partner $i \quad(i=1,2, \ldots, n)$;

$a_{i} \in[0,+\infty)$, the productive effort of partner $i$;

$C_{i}\left(a_{i}\right)$, the cost function of partner $i$; strictly increasing convex differentiable function, and $C_{i}(0)=0$;

$a=\left(a_{1}, a_{2}, \ldots, a_{n}\right)$, the vector of all partners' productive efforts;

$x(a)$, the common output decided by $a$, strictly increasing concave differentiable function and $x(0)=0$;

$\pi(a, \theta)$, monetary income (outputs);

$f\left(\pi, a_{1}, a_{2}, \ldots, a_{n}\right)$, the probability density function of $\pi$

$\rho_{A_{i}}$, the partner $i$ 's risk aversion level;

$s_{i}(\pi)$, the revenue sharing factor of the partner.

As the owner can observe the partners' actions, the owner doesn't need to incentive the partners, its objective is to maximize the utility of its own profit by allocating the total revenue from the VE project including choosing $s_{i}(\pi)(i=1,2, \ldots, n)$. Similar to subsection 1 , the model is presented as below:

$$
\begin{aligned}
& \max _{s_{1}(\pi), s_{2}(\pi), \ldots, s_{n}(\pi)}\left\{E\left(v\left(\pi-\sum_{i=1}^{n} s_{i}(\pi)\right)\right)\right\} \\
& =\int v\left(\pi-\sum_{i=1}^{n} s_{i}(\pi)\right) f\left(\pi, a_{1}, a_{2}, \ldots, a_{n}\right) d \pi
\end{aligned}
$$

Such that

$$
\begin{aligned}
& \int u_{i}\left(s_{i}(\pi)\right) f\left(\pi, a_{1}, a_{2}, \ldots, a_{n}\right) d \pi-C_{i}\left(a_{i}\right) \geq \overline{u_{i}} \\
& \quad(i=1,2, \ldots, n)
\end{aligned}
$$

Again, we construct the Lagrangian function as follows:

$$
\begin{aligned}
& L\left(s_{1}(\pi), s_{2}(\pi), \ldots, s_{n}(\pi)\right) \\
& =\int v\left(\pi-\sum_{i=1}^{n} s_{i}(\pi)\right) f\left(\pi, a_{1}, a_{2}, \ldots, a_{n}\right) d \pi \\
& \quad+\sum_{i=1}^{n} \lambda_{i}\left(\int u_{i}\left(s_{i}(\pi)\right) f\left(\pi, a_{1}, a_{2}, \ldots, a_{n}\right) d \pi-C_{i}\left(a_{i}\right)-\overline{u_{i}}\right)
\end{aligned}
$$

$\lambda_{i}(i=1,2, \ldots, n)$ are the Lagrange multipliers and are strictly positive constants in (21). We then consider the first-order condition as follow:

$$
\left\{\begin{array}{c}
\frac{\partial L}{\partial s_{i}}=-v^{\prime}\left(\pi-\sum_{i=1}^{n} s_{i}(\pi)\right)+\lambda_{i} u_{i}^{\prime}\left(s_{i}(\pi)\right)=0(i=1,2, \ldots, n) \\
\lambda_{i}=\frac{v^{\prime}\left(\pi-\sum_{i=1}^{n} s_{i}(\pi)\right)}{u_{i}^{\prime}\left(s_{i}(\pi)\right)} \equiv \frac{v^{\prime}}{u_{i}^{\prime}}(i=1,2, \ldots, n) \\
-v^{\prime \prime}\left(1-\frac{d s_{i}^{*}}{d \pi}\right)+\lambda_{i} u_{i}^{\prime \prime} \frac{d s_{i}^{*}}{d \pi}=0(i=1,2, \ldots, n)
\end{array}\right.
$$

Combining the above equations, we have

$$
\frac{d s_{i}^{*}}{d \pi}=\frac{\rho_{P}}{\rho_{A_{i}}+\rho_{P}}, \quad(i=1,2, \ldots, n)
$$

Such that

$$
\rho_{P}=-\frac{v^{\prime \prime}}{v^{\prime}} \text { and } \rho_{A_{i}}=-\frac{u_{i}^{\prime \prime}}{u_{i}^{\prime}},(i=1,2, \ldots, n)
$$

We assume that

$$
\frac{d s_{i}^{*}}{d \pi}=\frac{\rho_{P}}{\rho_{A_{i}}+\rho_{P}}=\beta_{i}(\pi),(i=1,2, \ldots, n)
$$

Then $s_{i}^{*}(\pi)=\alpha_{i}+\int_{0}^{\pi} \beta_{i}(t) d t, \quad(i=1,2, \ldots, n)$ 
We note if $\rho_{P}$ and $\rho_{A_{i}}(i=1,2, \ldots, n)$ are constants (no relation with their levels of income), the optimal contract is linear, i.e.

$$
s_{i}^{*}(\pi)=\alpha_{i}+\beta_{i} \pi
$$

Now, the improved risk programming model is given as follows:

$$
\min _{\beta_{1}, \beta_{2}, \ldots, \beta_{n}}\left\{\Delta R C=\frac{1}{2} \sigma^{2} \sum_{i=1}^{n} \rho_{i} \beta_{i}^{2}\right\}
$$

Such that

$$
\begin{aligned}
& \int u_{i}\left(s_{i}(\pi)\right) f\left(\pi, a_{1}, a_{2}, \ldots, a_{n}\right) d \pi-C_{i}\left(a_{i}\right) \geq \overline{u_{i}} \\
& (i=1,2, \ldots, n) \\
& \int u_{i}\left(s_{i}(\pi)\right) f_{a_{i}}\left(\pi, a_{1}, a_{2}, \ldots, a_{n}\right) d \pi=C_{i}^{\prime}\left(a_{i}\right) \\
& (i=1,2, \ldots, n) \\
& \Delta R C_{i} \leq R_{i}, \quad(i=1,2, \ldots, n) \\
& \sum_{i=1}^{n} \Delta R C_{i} \leq R \\
& \left(\beta_{1}, \beta_{2}, \ldots, \beta_{n}\right)=\arg \max \left\{E\left(v\left(\pi-\sum_{i=1}^{n} s_{i}(\pi)\right)\right)\right\}
\end{aligned}
$$

\subsection{The Optimal Contract of Risk Allocation with Asymmetric Information to Multiple Partners}

We then discuss the case of risk allocation with asymmetric information involving an owner and $n$ risk-averse partners in a VE. As the partners' action can't be observed, the owner has to incentive to prevent the partners from free riding. So the incentive constraints are necessary. The owner's objective is to maximize the utility of its own profit by allocating the total revenue from the VE project including choosing $s_{i}(\pi)$ and incentive the partners $(i=1,2, \ldots, n)$. The model is given as follows:

$$
\begin{aligned}
& \max _{s_{1}(\pi), s_{2}(\pi), \ldots, s_{n}(\pi)}\left\{E\left(v\left(\pi-\sum_{i=1}^{n} s_{i}(\pi)\right)\right)\right\} \\
& =\int v\left(\pi-\sum_{i=1}^{n} s_{i}(\pi)\right) f\left(\pi, a_{1}, a_{2}, \ldots, a_{n}\right) d \pi
\end{aligned}
$$

Such that

$$
\begin{aligned}
& \int u_{i}\left(s_{i}(\pi)\right) f\left(\pi, a_{1}, a_{2}, \ldots, a_{n}\right) d \pi-C_{i}\left(a_{i}\right) \geq \overline{u_{i}} \\
& (i=1,2, \ldots, n) \\
& \int u_{i}\left(s_{i}(\pi)\right) f_{a_{i}}\left(\pi, a_{1}, a_{2}, \ldots, a_{n}\right) d \pi=C_{i}^{\prime}\left(a_{i}\right) \\
& \quad(i=1,2, \ldots, n)
\end{aligned}
$$

We then construct the Lagrangian function:

$$
\begin{aligned}
& L\left(s_{1}(\pi), s_{2}(\pi), \ldots, s_{n}(\pi)\right) \\
& =\int v\left(\pi-\sum_{i=1}^{n} s_{i}(\pi)\right) f\left(\pi, a_{1}, a_{2}, \ldots, a_{n}\right) d \pi \\
& \quad+\sum_{i=1}^{n} \lambda_{i}\left(\int u_{i}\left(s_{i}(\pi)\right) f\left(\pi, a_{1}, a_{2}, \ldots, a_{n}\right) d \pi\right. \\
& \left.\quad-C_{i}\left(a_{i}\right)-\overline{u_{i}}\right) \\
& +\sum_{i=1}^{n} \mu_{i}\left(\int u_{i}\left(s_{i}(\pi)\right) f_{a_{i}}\left(\pi, a_{1}, a_{2}, \ldots, a_{n}\right) d \pi\right. \\
& \left.\quad-C_{i}^{\prime}\left(a_{i}\right)\right)
\end{aligned}
$$

where $\lambda_{i}$ and $\mu_{i}(i=1,2, \ldots, n)$ are the Lagrangian multipliers of participation constraints and the incentive constraints respectively. The optimal conditions are given as below:

$$
\frac{v^{\prime}\left(\pi-\sum_{i=1}^{n} s_{i}(\pi)\right)}{u_{i}^{\prime}\left(s_{i}(\pi)\right)}=\lambda_{i}+\mu_{i} \frac{f_{a_{i}}\left(\pi, a_{1}, a_{2}, \ldots, a_{n}\right)}{f\left(\pi, a_{1}, a_{2}, \ldots, a_{n}\right)}
$$

Compared with (21), it shows that, if the owner cannot observe $a$, the Pareto efficiency risk allocation is impossible. The partners have to bare more risks.

\section{A Specific Example}

In this section, in order to have a better understanding of our models in section 3, we process example analyses to make further investigation. To simplify the analysis, we employ Linear sharing rules, Exponential utility, and normally distributed random variables in this paper, i.e., adopt agency model developed by Holmstrom and Milgrom [20] which has been proved to be much more tractable in addressing multi-action and multi-period models. This assumption does not affect the core issue, and the total output of the VE is assumed to be a linear function of the partners' productive efforts, which is extended from the simple model Holmstrom and Milgrom (1987) proposed. The total output of the VE is:

$$
\pi=\sum_{i=1}^{n} a_{i}+\theta \text {, and } \theta \text { subjects to normal distribution }
$$
$N\left(0, \sigma^{2}\right)$.

Therefore $E(\pi)=\sum_{i=1}^{n} a_{i}, \operatorname{Var}(\pi)=\sigma^{2}$

Then $s_{i}(\pi)=\alpha_{i}+\beta_{i} \pi, \quad(i=1,2, \ldots, n)$

And $s(\pi)=\sum_{i=1}^{n} s_{i}(\pi)=\sum_{i=1}^{n} \alpha_{i}+\pi \sum_{i=1}^{n} \beta_{i}$

The owner's expected utility is given by

$$
E\left(v\left(\pi-\sum_{i=1}^{n} s_{i}(\pi)\right)\right)=-\sum_{i=1}^{n} \alpha_{i}+\pi\left(1-\sum_{i=1}^{n} \beta_{i}\right)
$$


It is assumed that the marginal cost is increasing in the level of effort and the cost function takes the quadratic form [21], to simplify the analysis, we assume that the cost function is continuously differentiable and strictly convex and take the form:

$$
C_{i}\left(a_{i}\right)=\frac{1}{2} b_{i} a_{i}^{2}
$$

And $b_{i}$ is the coefficient (marginal cost). Partner $i$ 's actual revenue is

$$
\omega_{i}=s_{i}(\pi)-C_{i}\left(a_{i}\right)=\alpha_{i}+\beta_{i}\left(\sum_{i=1}^{n} a_{i}+\theta\right)-\frac{1}{2} b_{i} a_{i}^{2}
$$

As the owner and every partner have constant absolute risk aversion, which implies its utility function is of the negative exponential form. Then, we make the usual transformation of expected utility into mean-variance terms as follows [22]:

$$
\begin{aligned}
& E\left(\omega_{i}\right)-\frac{1}{2} \rho_{i} \beta_{i}^{2} \sigma^{2} \\
& =\alpha_{i}+\beta_{i} \sum_{i=1}^{n} a_{i}-\frac{1}{2} \rho_{i} \beta_{i}^{2} \sigma^{2}-\frac{1}{2} b_{i} a_{i}^{2}
\end{aligned}
$$

And $\frac{1}{2} \rho_{i} \beta_{i}^{2} \sigma^{2}$ is partner $i$ 's risk cost. If $a=$ $\left(a_{1}, a_{2}, \ldots, a_{n}\right)$ can be observed, the owner can decide $\left(\alpha_{i}, \beta_{i}, a_{i}\right)$. The model is given as follows:

$$
\max _{\left(\alpha_{i}, \beta_{i}, a_{i}\right)}\left\{E(v)=-\sum_{i=1}^{n} \alpha_{i}+\pi\left(1-\sum_{i=1}^{n} \beta_{i}\right)\right\}
$$

Such that

$$
\begin{aligned}
& \alpha_{i}+\beta_{i}\left(\sum_{i=1}^{n} a_{i}+\theta\right)-\frac{1}{2} \rho_{i} \beta_{i}^{2} \sigma^{2}-\frac{1}{2} b_{i} a_{i}^{2} \geq \overline{\omega_{i}} \\
& (i=1,2, \ldots, n)
\end{aligned}
$$

$\overline{\omega_{i}}$ is the reservation utility. The maximization problem can be formulated as:

$$
\max _{\left(\beta_{i}, a_{i}\right)}\left\{f=a-\frac{1}{2} \sigma^{2} \sum_{i=1}^{n} \rho_{i} \beta_{i}^{2}-\frac{1}{2} \sum_{i=1}^{n} b_{i} a_{i}^{2}-\sum_{i=1}^{n} \overline{\omega_{i}}\right\}
$$

The optimality conditions are

$$
\begin{array}{r}
\frac{\partial f}{\partial \beta_{i}}=-\rho_{i} \beta_{i} \sigma^{2}=0, \frac{\partial f}{\partial a_{i}}=1-b_{i} a_{i}=0 \\
(i=1,2, \ldots, n) \\
\text { i.e., } a_{i}^{*}=\frac{1}{b_{i}}, \quad \beta_{i}^{*}=0 \text { and } \alpha_{i}^{*}=\overline{\omega_{i}}+\frac{1}{2 b_{i}}
\end{array}
$$

The Pareto efficiency risk allocation requires the partners to bear no risk $\left(\beta_{i}^{*}=0\right)$. If $a=\left(a_{1}, a_{2}, \ldots, a_{n}\right)$ can- not be observed, the owner can decide $\left(\alpha_{i}, \beta_{i}\right)$.The partners choose the action $a$ to maximize their expected utility:

$$
\max _{a_{1}, a_{2}, \ldots, a_{n}} \int u_{i}\left(s_{i}(\pi)\right) f\left(\pi, a_{1}, a_{2}, \ldots, a_{n}\right) d \pi-C_{i}\left(a_{i}\right)
$$

The partial derivative with respect to $\pi$ is

$$
\begin{aligned}
& \int u_{i}\left(s_{i}(\pi)\right) f_{a_{i}}\left(\pi, a_{1}, a_{2}, \ldots, a_{n}\right) d \pi=C_{i}^{\prime}\left(a_{i}\right) \\
& \quad(i=1,2, \ldots, n)
\end{aligned}
$$

Since $\beta_{i}=b_{i} a_{i}, \quad a_{i}=\frac{\beta_{i}}{b_{i}} \quad(i=1,2, \ldots, n)$ the problem can be transformed into the following form

$$
\max _{\left(\alpha_{i}, \beta_{i}\right)}\left\{f=-\sum_{i=1}^{n} \alpha_{i}+a\left(1-\sum_{i=1}^{n} \beta_{i}\right)\right\}
$$

Such that

$$
\begin{aligned}
& \alpha_{i}+\beta_{i}\left(\sum_{i=1}^{n} a_{i}+\theta\right)-\frac{1}{2} \rho_{i} \beta_{i}^{2} \sigma^{2}-\frac{1}{2} b_{i} a_{i}^{2} \geq \overline{\omega_{i}} \\
& \quad(i=1,2, \ldots, n) \\
& a_{i}=\frac{\beta_{i}}{b_{i}} \quad(i=1,2, \ldots, n)
\end{aligned}
$$

The problem can then be further transformed to the following problem:

$$
\begin{aligned}
& \max _{\beta_{i}}\left\{f=\sum_{i=1}^{n} \frac{\beta_{i}}{b_{i}}-\frac{1}{2} \sigma^{2} \sum_{i=1}^{n} \rho_{i} \beta_{i}^{2}-\frac{1}{2} \sum_{i=1}^{n} \frac{\beta_{i}^{2}}{b_{i}}-\sum_{i=1}^{n} \overline{\omega_{i}}\right\} \\
& \frac{\partial f}{\partial \beta_{i}}=\frac{1}{b_{i}}-\rho_{i} \beta_{i} \sigma^{2}-\frac{\beta_{i}}{b_{i}}=0 \quad(i=1,2, \ldots, n)
\end{aligned}
$$

Here we note that

$$
\beta_{i}=\frac{1}{1+\rho_{i} b_{i} \sigma^{2}}>0, \quad(i=1,2, \ldots, n)
$$

This also means that the partners must bear certain risk. While we can see $\beta_{i}$ is a deceasing function in $\rho_{i}, b_{i}$ and $\sigma^{2}$. In other words, the risks the partners bear are negatively correlated to their risk aversion levels and the output variances. Now partner $i$ 's risk costs is given by

$$
\begin{aligned}
& \Delta R C_{i}=\frac{1}{2} \sigma^{2} \rho_{i} \beta_{i}^{2}=\frac{\rho_{i} \sigma^{2}}{2\left(1+\beta_{i} b_{i} \sigma^{2}\right)^{2}}>0 \\
& (i=1,2, \ldots, n)
\end{aligned}
$$

\section{Multi-principal Models}

In this section, we extend the principal-agent framework with risk-neutral principals to situations in which several principals simultaneously and independently attempt to influence a common agent that is considering the case of 
multi principal agency relationships of the members in VE which involving $n$ risk-neutral principals and a risk-aversion agent. We analyze the moral hazard and give optimal contract. To facilitate our discussion, we define the following notations and impose the following assumptions.

$N=\{1,2, \ldots, n\}$, the set of principals;

$a_{i}$, the productive effort of the agent to the principal $i$;

$M$, the upper bound productive effort of the agent to the principals;

$\theta$, the uncertain variance the agent can't control, and it subjects to normal distribution $N\left(0, \sigma^{2}\right)$;

$\pi_{i}=\pi_{i}\left(a_{i}, \theta\right)$, the monetary income (outputs) of the effort $a_{i}$;

$s_{i}=s_{i}\left(\pi_{i}\right)$, the incentive contract (a way to repay the agent with respect to $a_{i}$ );

$C\left(a_{i}\right)$, the cost function of efforts $a_{i}$;

$v_{i}(x)=v\left(\pi_{i}-s_{i}\left(\pi_{i}\right)\right)$, the principal $i$ 's utility function

$u(x)=\sum_{i=1}^{n} u_{i}\left(s_{i}\left(\pi_{i}\right)-C\left(a_{i}\right)\right)$, the agent's utility function respectively;

$\omega_{i}$, the actual profit from principal $i$;

$\varpi_{i} \geq 0$, the opportunities income (reservation income) of that the principal $i$ guarantees;

In the multi-principal model, we assume that the total productive effort of the agent has a limited $M$, which means the resources are limited and guarantees the boundedness of the solution. Because there are multiple principals, the model becomes more complex. As the relationship between the principals may affect the results of the model, we consider the competition relationships (non-cooperative). The model is given as follows: Every principal give a $s_{i}\left(\pi_{i}\right)$ non-cooperatively, the agent's objective is to maximize the utility of its own total profit by allocating the total revenue from the VE project including choosing every $a_{i}, \quad(i=1,2, \ldots, n)$ :

$$
\begin{aligned}
& \max _{s_{i}(\pi)}\left\{E\left(v\left(\pi_{i}-s_{i}\left(\pi_{i}\right)\right)\right\}\right. \\
& \text { s.t. }(I R) u_{i}=E\left(u_{i}\left(s_{i}\left(\pi_{i}\right)\right)\right)-C\left(a_{i}\right) \geq \varpi_{i} \\
& \quad(i=1,2, \ldots, n) \\
& (I C) \max u=\sum_{i=1}^{n} u_{i} \\
& \sum_{i=1}^{n} a_{i} \leq M
\end{aligned}
$$

In order to have a better understanding of the multiprincipal models in this section, we process example analyses to make further investigation. To simplify the analysis, we employ Linear sharing rules, Exponential utility, i.e., adopt agency model developed by Holmstrom and Milgrom [20] which has been proved to be much more tractable in addressing multi-action and multi-period models, and consider the case of two principals. This assumption does not affect the core issue. We add the following assumptions:

$k_{i}>0$, the agent to principal $i$ 's effort level to the impact factor of the marginal output;

$\pi_{i}=\pi_{i}\left(a_{i}, \theta\right)=k_{i} a_{i}+\theta$, the output of the agent to principal $i$;

$\alpha_{i}$, the fixed remuneration of member enterprise $i$;

$\beta_{i} \geq 0$, the revenue sharing factor of member enterprise $i$;

$\rho$, the agent's risk aversion level;

$C\left(a_{i}\right)=\frac{1}{2} b a_{i}^{2}$, the cost of $a_{i} ;$

$s_{i}(\pi)=\alpha_{i}+\beta_{i} \pi$, linear sharing rules;

$$
\omega_{i}=\alpha_{i}+\beta_{i} \pi-\frac{1}{2} b_{i} a_{i}^{2} .
$$

For principal 1,

$$
\begin{aligned}
& \max _{\alpha_{1}, \beta_{1}}\left\{v_{1}=E\left(v_{1}\left(\pi_{1}-s_{1}\left(\pi_{1}\right)\right)\right)=-\alpha_{1}+\left(1-\beta_{1}\right) k_{1} a_{1}\right\} \\
& \text { s.t. }(I R) u_{1}=\alpha_{1}+\beta_{1} k_{1} a_{1}-\frac{1}{2} b a_{1}^{2}-\frac{1}{2} \rho \beta_{1}^{2} \sigma^{2} \geq \varpi_{1} \\
& (I C) \max u=u_{1}+u_{2} \\
& a_{1}+a_{2} \leq M
\end{aligned}
$$

For principal 2,

$$
\begin{aligned}
& \max _{\alpha_{2}, \beta_{2}}\left\{v_{2}=E\left(v_{2}\left(\pi_{2}-s_{2}\left(\pi_{2}\right)\right)\right)=-\alpha_{2}+\left(1-\beta_{2}\right) k_{2} a_{2}\right\} \\
& \text { s.t. }(I R) u_{2}=\alpha_{2}+\beta_{2} k_{2} a_{2}-\frac{1}{2} b a_{2}^{2}-\frac{1}{2} \rho \beta_{2}^{2} \sigma^{2} \geq \varpi_{2} \\
& \text { (IC) } \max u=u_{1}+u_{2} \\
& a_{1}+a_{2} \leq M
\end{aligned}
$$

To solve the above models, we construct the Lagrangian function as follows:

$$
\begin{aligned}
L= & u_{1}+u_{2}+\lambda\left(a_{1}+a_{2}-M\right) \\
= & \alpha_{1}+\beta_{1} k_{1} a_{1}-\frac{1}{2} b a_{1}^{2}-\frac{1}{2} \rho \beta_{1}^{2} \sigma^{2} \\
& +\alpha_{2}+\beta_{2} k_{2} a_{2}-\frac{1}{2} b a_{2}^{2}-\frac{1}{2} \rho \beta_{2}^{2} \sigma^{2} \\
& +\lambda\left(a_{1}+a_{2}-M\right)
\end{aligned}
$$

$\lambda$ is the Lagrange multiplier. We then consider the first-order condition as follow: 


$$
\left\{\begin{array}{c}
\frac{\partial L}{\partial a_{1}}=\beta_{1} k_{1}-b a_{1}+\lambda=0 \\
\frac{\partial L}{\partial a_{2}}=\beta_{2} k_{2}-b a_{2}+\lambda=0 \\
\frac{\partial L}{\partial \lambda}=a_{1}+a_{2}-M=0
\end{array}\right.
$$

So the optimal productive effort is

$$
\left\{\begin{array}{l}
a_{1}^{*}=\frac{M}{2}+\frac{\beta_{1} k_{1}-\beta_{2} k_{2}}{2 b} \\
a_{2}^{*}=\frac{M}{2}+\frac{\beta_{2} k_{2}-\beta_{1} k_{1}}{2 b}
\end{array}\right.
$$

Substituting $a_{1}^{*}, a_{2}^{*}$ into the principal 1 's object function, the optimal problem is:

$$
\begin{aligned}
\max _{\beta_{1}}\left\{v_{1}=k_{1} a_{1}-\frac{1}{2} b a_{1}^{2}-\frac{1}{2} \rho \beta_{1}^{2} \sigma^{2}-\varpi_{1}\right\} \\
=k_{1}\left(\frac{M}{2}+\frac{\beta_{1} k_{1}-\beta_{2} k_{2}}{2 b}\right)-\frac{1}{2} b\left(\frac{M}{2}+\frac{\beta_{1} k_{1}-\beta_{2} k_{2}}{2 b}\right)^{2} \\
-\frac{1}{2} \rho \beta_{1}^{2} \sigma^{2}-\varpi_{1}
\end{aligned}
$$

The first-order condition on the $\beta_{1}$ :

$$
\frac{\partial v_{1}}{\partial \beta_{1}}=0
$$

Then the optimal solution is

$$
\left\{\begin{array}{c}
\beta_{1}^{*}=\frac{2 k_{1}^{2}-k_{1} b M+k_{1} k_{2} \beta_{2}}{k_{1}^{2}+4 b \rho \sigma^{2}} \\
\alpha_{1}^{*}=\beta_{1} k_{1} a_{1}-\frac{1}{2} b a_{1}^{2}-\frac{1}{2} \rho \beta_{1}^{2} \sigma^{2}-\varpi_{1}
\end{array}\right.
$$

Similarly, we can solve the optimal solution to the principal 2:

$$
\left\{\begin{array}{c}
\beta_{2}^{*}=\frac{2 k_{2}^{2}-k_{2} b M+k_{1} k_{2} \beta_{1}}{k_{2}^{2}+4 b \rho \sigma^{2}} \\
\alpha_{2}^{*}=\beta_{2} k_{2} a_{2}-\frac{1}{2} b a_{2}^{2}-\frac{1}{2} \rho \beta_{2}^{2} \sigma^{2}-\varpi_{2}
\end{array}\right.
$$

From the above $a_{1}^{*}, a_{2}^{*}, \beta_{1}^{*}$ and $\beta_{2}^{*}$, we can analysis their mutual relationships and conclude that. The decision-making influences each other, when the other conditions had been given; the efforts of the agent depend on the strength of incentives of all principals. And the intensity of incentives weakens each other. These hypothesizes are also supported by many realistic cases.

\section{Conclusions}

Virtual enterprise is the main form of cooperation between enterprises today. Researching on the risk alloca- tion in VE has both theoretical and practical importance. On the basis of the introduction of the concepts of risk analysis, this paper mainly describes the risk allocation of VE based on the principal-agent theory and draws the following conclusions: if the owner cannot observe the partners' efforts level, the Pareto efficiency risk allocation is impossible to achieve. In other words, the partners must bear certain risks, and the risks the partners bearing are negatively correlated to his risk aversion level and the output variance. For the perfection of the problem, we consider the case of multiple principal based on common agency in Section 5. To simplify the analysis and explore the implications of the risk allocation mechanism, we have made some restrictions to the example in section 4, such as linear/quadratic forms, independence, normal distribution, etc. In the future research, we will relax these restrictions to investigate the allocation mechanism under much more general environment, and consider the incentive mechanism when the relationship between the principals is cooperative.

\section{Acknowledgements}

The authors wish to thank the support of the National Natural Science Foundation of China under Grant No. 70671020, No. 70721001, No. 70931001 and No. 60673159, Specialized Research Fund for the Doctoral Program of Higher Education under Grant No. 20070145017, the Fundamental Research Funds for the Central Universities under Grant No. N090504006 and No. N090504003, Science and Technology Research Fund of Bureau of Education of Liaoning Province, and the RGC Grant 7017/07P, HKU CRCG Grants, Hung Hing Ying Physical Sciences Research Fund and HKU Strategic Research Theme Fund on Computational Sciences.

\section{REFERENCES}

[1] M. T. Martinez, P. Fouletier, K. H. Park and J. Favrel, "Virtual Enterprise-Organization, Evolution and Control," International Journal of Production Economics, Vol. 74, No. 3, 2001, pp. 225-238.

[2] A. Mowshowitz, "Virtual Organization," Communication of the ACM, Vol. 40, No. 9, 1997, pp. 30-37.

[3] M. Ojala and J. Hallikas, "Investment Decision-Making in Supplier Networks: Management of Risk," International Journal of Production Economics, Vol. 104, No. 1, 2006, pp. 201-213.

[4] Q. L. Gao and G. P. Cheng, "Virtual Enterprise's Operation Risk," Value Engineering, Vol. 104, No. 9, 2006, pp. 104-105.

[5] M. Thomas and E. Norman, "Moral Hazards on the Road to the 'Virtual' Corporation," Business Ethics Quarterly, Vol. 8, No. 2, 1998, pp. 273-292.

[6] M. Gaynor and P. Gertle, "Moral Hazard and Risk Spreading in Partnerships," Journal of Economics, Vol. 26, No. 4, 1995, pp. 591-613. 
[7] F. Ye and Y. N. Li, "Group Multi-Attribute Decision Model to Partner Selection in the Formation of Virtual Enterprise under Incomplete Information," Expert Systems with Applications, Vol. 36, No. 5, 2009, pp. 9350-9357.

[8] A. H. Yannis and C. G. Andreas, "A Goal Programming Model for Partner Selection Decisions in International Joint Ventures," Journal of Operations Management, Vol. 138, No. 3, 2002, pp. 649-662.

[9] H. M. Gou, B. H. Huang, W. H. Liu and X. Li, "A Framework for Virtual Enterprise Operation Management," Computers in Industry, Vol. 50, No. 3, 2003, pp. 333-352.

[10] A. F. Cutting-Decelle, R. I. M. Young and B. P. Das, "Information Exchanges in a Cross Disciplinary Supply Chain: Formal Strategy and Application," INCOM'06 Conference, Saint-Etienne, 2006.

[11] W. Ip, M. Huang, K. Yung and D. Wang, "Genetic Algorithm Solution for a Risk-Based Partner Selection Problem in a Virtual Enterprise," Computers and Opera tions Research, Vol. 30, No. 2, 2003, pp. 213-231.

[12] H. Lars, "Managing Cooperative Research and Development: Partner Selection and Contract Design," $R \& D$ Management, Vol. 23, No. 4, 2007, pp. 273-285.

[13] L. M. Camarinha-Matos and C. Lima, "Cooperation Coordination in Virtual Enterprises," Journal of Intelligent Manufacturing, Vol. 12, No. 2, 2001, pp. 133-150.

[14] R. Narasimhan and T. P. Srinivas, "Perspectives on Risk
Management in Supply Chains," Journal of Operations Management, Vol. 27, No. 21, 2009, pp. 114-118.

[15] L. H. Sung, H. P. Yeng, M. R. Yan and J. R. Lee, "On-Line Multi-Criterion Risk Assessment Model for Construction Joint Ventures in China," Automation in Construction, Vol. 16, No. 5, 2007, pp. 607-619.

[16] B. Holmstrom, "Moral Hazard and Observability," Bell journal of Economics, Vol. 10, No. 1, 1979, pp. 74-91.

[17] G. Esther, "Multi Principal Agency Relationships as Implied by Product Market Competition," Journal of Economic \& Management Strategy, Vol. 6, No. 1, 2004, pp. 235-256.

[18] B. D. Bernheim and M. D. Whinston, "Common Agency," Econometrica, Vol. 54, No. 4, 1986, pp. 923-942.

[19] A. Attar, E. Campioni, G. Piaser and U. Rajan, "On Multiple-Principal Multiple-Agent Models of Moral Hazard," Games and Economic Behavior, Vol. 68, No. 1, 2010, pp. 376-380.

[20] L. M. Camarinha-Matos and C. Lima, "Aggregation and Linearity in the Provision of Intertemporal Incentives," Econometrica, Vol. 55, No. 5, 1987, pp. 303-328.

[21] G. Feltham and J. Xie, "Performance Measure Congruity and Diversity in Multi-Task Principal-Agent Relations," The Accounting Review, Vol. 69, No. 3, 1994, pp. 429-453.

[22] R. A. Lambert, "Contracting Theory and Accounting," Journal of Accounting and Economics, Vol. 32, No. 1-3, 2001, pp. 3-87. 